\title{
COLUMN: Core Less Unformed Machine
}

Yasutaka Takeda, Kohei Yoshida, Shotaro Baba, P. Ravindra S De Silva, and Michio Okada

Interactions and Communication Design Lab, Toyohashi University of Technology Toyohashi 441-8580, JAPAN

\{takeda, yoshida, baba\}@icd.cs.tut.ac.jp, \{ravi, okada\}@tut.jp

http://www.icd.cs.tut.ac.jp/en/profile.html

\section{Introduction}

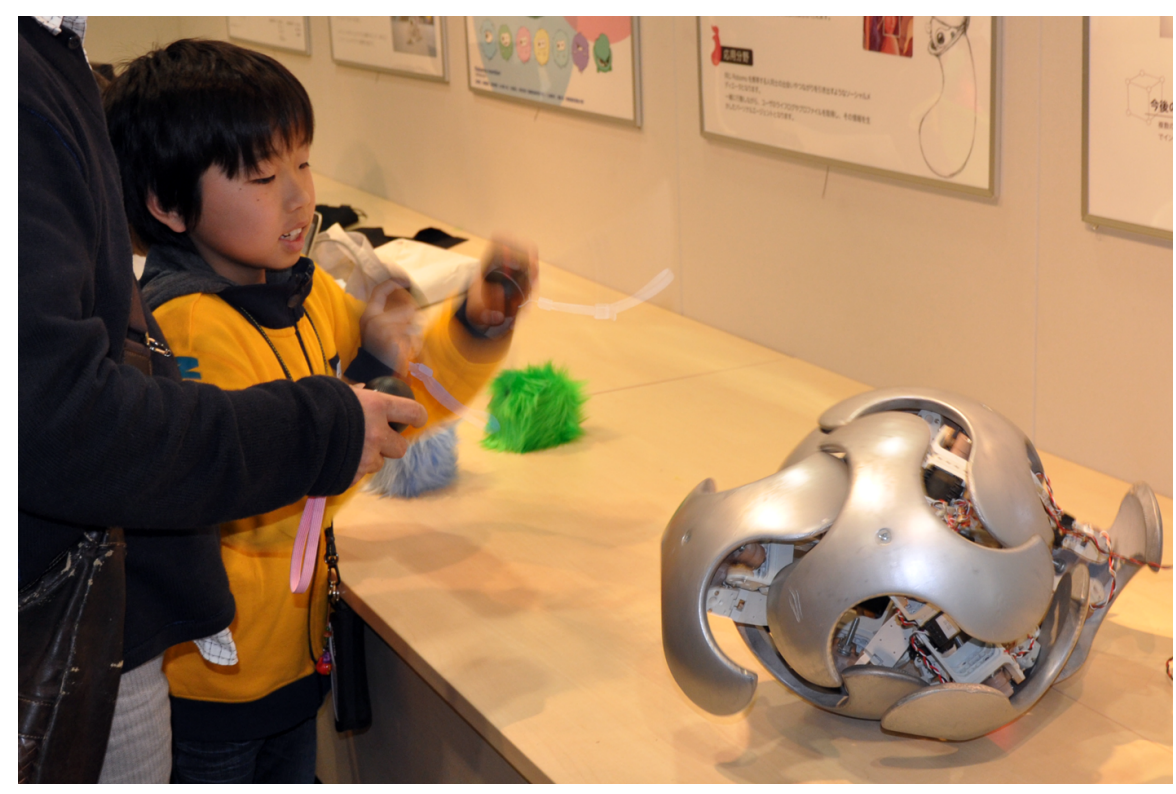

Fig. 1. Appearance of Core Less Unformed Machine (COLUMN).

The concept of a self-configurable (transformable) robot is versatile and effective in space applications, entertainment, natural disasters, and developing and controlling such robots is really challengeable [1]. Exist mechanism are attempting to connect their modules as external parts, as well as try to maintain their structural shape as snake or walker [2][3]. Always challengeable to design the motion mechanism and autonomous capability for such kind of novel architectural artifact which is entirely different from the existing. 
In this video, we presented a transformable artifact (COLUMN) which becomes a social mediator in the proposed context. The COLUMN is a soccer ball-shaped interactive artifact consisting of eight modules that are connected to the twelve servomotors (Figure 1). We can transfer the COLUMN body shape by moving the actuators with modules, and these actuators can be controlled by a wireless communicator called a "COLUMN Gear." Each of the users (three participants) can control the 4 servo-motors of the COLUMN, and a user can swing the COLUMN gear to change its body shape (transfer its modules). Users have to coordinate with each other to boost its transformable behaviors while preserve their co-action in the dynamic interactions. In contrast to the previous utilization of robot as a social mediator [4][5], in this study interactive users have to understand what/how they are controlling the robot, and necessitate inferring the way of connecting with others, and also understand essential in-time coordinate in the dynamic interaction. This type of synchronization with each other's swing (toward achieving their goal) can be defined as the interpersonal coordination in the context. Therefore, by using the patterns of interpersonal coordination, our motivation is to explore users invent transformable behaviors for the COLUMN.

\section{Acknowledgement}

This research has been supported by both Grant-in-Aid for scientific research of KIBAN-B (26280102) and Grant-in-Aid for scientific research for HOUGA (2465- 0053) from the Japan Society for the Promotion of Science (JSPS).

\section{References}

1. M. Yim, W.M. Shen, B. Salemi, D. Rus, M. Moll, H. Lipson, E. Klavins, and G. S. Chirikjian. Modular self-reconfigurable robot systems - challenges and opportunities for the future. IEEE Robotics and Automation Magazine, 45-53, 2007.

2. M. Yim, D. Goldberg, and A. Casal. Connectivity planning for closed-chain reconfiguration Proceedings of the SPIE Vol 4196, Sensor fusion and decentralized control in robotics systems., 402-412, 2002.

3. P. Will, and A. Castano. Representing and discovering the configuration of Conro robots Proceedings of the 2001 IEEE International Conference on Robotics \& Automation (ICRA),, 3503-3509, 2001.

4. F. Papadopoulos, K. Dautenhahn, and W. C. Ho. Exploring the use of robots as social mediators in a remote human-human collaborative communication experiment. Paladyn, 3(1):1-10, 2012.

5. B. Robins, K. Dautenhahn, R. Boekhorst, and A. Billard Robotic assistants in therapy and education of children with autism: Can a small humanoid robot help encourage social interaction skills? Universal Access in the Information Society, 4(2):105-120, 2005. 